\title{
Review Article \\ Uveitis in the Aging Eye: Incidence, Patterns, and Differential Diagnosis
}

\author{
Marwan R. Abdulaal, Bachir H. Abiad, and Rola N. Hamam \\ Department of Ophthalmology, American University of Beirut Medical Center, P.O. Box 11-0236/D41, Riad El Solh, \\ Beirut 11072020, Lebanon \\ Correspondence should be addressed to Rola N. Hamam; rh46@aub.edu.lb
}

Received 16 March 2015; Accepted 27 April 2015

Academic Editor: Farzin Forooghian

Copyright (C) 2015 Marwan R. Abdulaal et al. This is an open access article distributed under the Creative Commons Attribution License, which permits unrestricted use, distribution, and reproduction in any medium, provided the original work is properly cited.

Uveitis is a vision threatening inflammation of the eye that carries considerable morbidity. It is responsible for $10 \%$ of legal blindness in the United States and up to $25 \%$ in the developing world. Uveitis in patients more than 60 years of age is less common. The aging body has a changing response of the immune system, which might reflect a different pattern of uveitis in the elderly population. In this paper we review the incidence and patterns of uveitis in the elderly as reported in the literature and discuss changes with time. We also delineate a thorough differential diagnosis of de novo uveitis in the elderly.

\section{Introduction}

Uveitis is inflammation of the middle-lining layer of the eye, comprising the iris, ciliary body, and choroid. It may involve other adjacent tissues, such as the retina, optic nerve, and vitreous humor [1]. This disease is a sight threatening condition worldwide. It accounts for up to $10 \%$ of legal blindness in United States and about 25\% in the developing countries $[2,3]$.

This disease is considered to affect young patients with median age at presentation in the third and fourth decade. This impression is based on epidemiology studies published in the 1960s, which demonstrated that uveitis occurs mainly in young adults at 20 to 50 years of age [1]. On the other hand, more recent reports from the United States showed significant increase in number of uveitis cases among elderly patients [4, 5].

According to World Population Aging report (2009), elderly or aged people are persons of 60 years of age or more [6]. Globally the population of older persons is growing considerably faster than the population as a whole, and it is expected to continue growing more rapidly than other age groups at least till 2050 [6]. Taking that into consideration, our knowledge of the prevalence of uveitis and its common types among elderly patients is essential to formulate and evaluate goals and programs and to enhance our understanding of this disease and prevent its long term complications.

The aim of our paper is to review uveitis epidemiology studies among elderly patients. Furthermore, we aim to discuss the pattern of presentation and diagnosis among elderly patients with uveitis and analyze any change over the past 5 decades. We also aim to delineate a thorough differential diagnosis of de novo uveitis in the elderly.

\section{Methods}

We performed an extensive literature search using the MEDLINE database, from 1964 to 2014. The search subject included uveitis: epidemiology, uveitis among elderly, etiology, classification, and diagnosis. The search was limited to the literature pertaining to humans, with no language limitation. Cross-referencing was also performed from the literature examined. An arbitrary method was used to select the studies to be included in our review, the emphasis being placed on obtaining studies that were representative of each region in different time era.

Data pertaining to age, gender, location of uveitis, chronicity, and diagnosis were extracted and analyzed in accordance with the International Uveitis Study Group (IUSG) recommendations [7]. Data from studies including 
adequate patient numbers spanning 5 decades to date was evaluated and analyzed.

\section{Results}

3.1. Epidemiology. Uveitis diagnosis among elderly patients older than 60 years of age was considered uncommon. The previous impression about the common age of presentation in uveitis was based on epidemiology studies in the last century. Darrell et al. in 1962 demonstrated that only 14\% of uveitis patients were considered elderly ( $>60$ years of age) [1]. However, this trend is changing especially in the developed countries in the last two decades $[4,5]$.

Reviewing the published uveitis epidemiology studies in the last 50 years, we find that the number of published reports has almost doubled after the year 2000 (15 reports published between 1960 and 1999 [1, 8-20] versus 26 reports published after 2000 [4, 5, 16, 20-43]). Specifically, much more reports have been published from developing countries after the year 2000 (3 reports were published prior to $2000[10,15,44]$ compared to 12 reports after 2000 [3, 21, 22, 24-26, 30, 32, 34, 35, 39, 41]).

Studying the total data from studies in the past 5 decades revealed that mean age at presentation is $38.0 \pm 5.0$ (range: 29.0-46.5) (Table 1). Comparing the mean age at presentation between developed versus developing countries shows that mean age at presentation in developed countries is more than in developing countries: $40.6 \pm 4.7$ (range: $33.8-46.1$ ) versus $34.4 \pm 2.7$ (range: $29.0-39.9$ ), respectively. If we compare data published before year 2000 to that published after, we find that there is no difference in the mean age at presentation (38.3 versus 37.8) (Table 1 ).

Also, comparing the mean proportion of elderly patients among total uveitis patients between developed versus developing countries revealed that this group of patients occupies a large number of patients in most of the epidemiology studies in developed countries with mean percentile of $18.6 \% \pm 5.7$ (range: 13.6-29.9\%). However, the mean percentile of elderly patients among uveitis patients in developing countries is limited to $7.2 \% \pm 2.2$ (range: $3.0-10.2 \%$ ) only. The percentile of elderly patients increased in reports from developing countries from 5.7 prior to year 2000 to $8.4 \%$ after. This might be due to increased reporting (Table 2).

This discrepancy in the mean percentile of elderly patients among total uveitis patients between developed and developing countries may have two potential explanations. First, most of the developed countries are considered according to the latest WHO report as aging countries with an increasing proportion of elderly population. Hence, more elderly patients with ocular inflammation are expected to be examined. Second, with an increasing awareness of the recommended screening tests and an improvement in the health care systems in the developed countries, more patients are expected to be examined and to be followed up in these countries including elderly patients.

Most of the major uveitis epidemiology studies demonstrated no gender preference or slight preference toward females. However, reviewing the data of eight epidemiology studies reporting the gender preference among elderly
TABLE 1: Mean age at presentation in uveitis epidemiology study.

\begin{tabular}{|c|c|c|}
\hline Study name & Total number of patients & Mean age \\
\hline Lebanon 2014 [21] & 209 & 36 \\
\hline Iran 2014 [22] & 2016 & 33.8 \\
\hline Italy 2010 [23] & 1065 & 41 \\
\hline Saudi Arabia 2010 [24] & 351 & 39.9 \\
\hline Colombia 2009 [25] & 693 & 31.7 \\
\hline Saudi Arabia 2009 [26] & 488 & 38 \\
\hline Japan 2009 [27] & 834 & 46.1 \\
\hline Japan 2009 (2) [28] & 1240 & 44.1 \\
\hline Germany 2009 [29] & 1916 & 35 \\
\hline Turkey 2008 [30] & 761 & 35.4 \\
\hline Thailand 2008 [31] & 200 & 38 \\
\hline Tunisia 2007 [32] & 219 & 34 \\
\hline China 2005 [33] & 1752 & 33.8 \\
\hline Turkey 2005 [34] & 300 & 35.7 \\
\hline Iran 2004 [35] & 544 & 33.1 \\
\hline Japan 2003 [36] & 189 & 45 \\
\hline USA 2003 [37] & 853 & 46.1 \\
\hline China 2003 [38] & 160 & 41.1 \\
\hline Saudi Arabia 2002 [39] & 200 & 34 \\
\hline Italy $2001[40]$ & 655 & 44.3 \\
\hline Cameron 2001 [41] & 38 & 33.9 \\
\hline India $2000[3]$ & 308 & 32.5 \\
\hline Italy 1996 [8] & 1417 & 30.7 \\
\hline UK 1996 [9] & 712 & 39.9 \\
\hline Sierra Leone 1996 [10] & 93 & 36 \\
\hline Japan 1997 [11] & 551 & 46.5 \\
\hline Switzerland 1994 [12] & 558 & 44 \\
\hline Holland 1992 [13] & 881 & 42 \\
\hline Portugal 1990 [14] & 450 & 36 \\
\hline Japan 1997 [11] & 407 & 40.7 \\
\hline Nigeria 1977 [15] & 1987 & 29 \\
\hline Mean & & 38.0 \\
\hline
\end{tabular}

patients revealed that uveitis among elderly females is significantly more than elderly males $(\mathrm{F}: \mathrm{M}=2.0)(P=0.021)$ $[11,12,14,27,28,38,42,45]$. The ratio decreased from 2.5 before 2000 to 1.7 after.

3.2. Most Common Location and Diagnosis. Generally, the most common location of uveitis worldwide is anterior uveitis. Similarly, most of the uveitis epidemiology studies in the elderly reported anterior uveitis as the most common location of this disease at presentation (507 out of 823 cases), followed by panuveitis (129 out of 823 cases) and posterior uveitis (112 out of 823 cases) (Table 3 ).

Also, the most common diagnosis of uveitis among elderly was reported as idiopathic uveitis in five out of ten reports. However, acute anterior uveitis was found to be the most common diagnosis in three studies. In addition, herpetic induced uveitis was reported as the second or the third most common cause of uveitis in elderly in seven out of ten studies. Most of the reviewed epidemiology studies 
TABLE 2: Proportion of elderly patients in uveitis epidemiology studies among developed countries.

\begin{tabular}{|c|c|c|}
\hline \multicolumn{3}{|c|}{ Developed countries } \\
\hline Name & Total number & Elderly patients percentile \\
\hline China 2012 [5] & 5866 & $1538(26.2 \%)$ \\
\hline Italy 2010 [23] & 1065 & $206(20.3 \%)$ \\
\hline Japan 2009 [27] & 843 & $224(26.8 \%)$ \\
\hline Japan 2009 (2) [28] & 1240 & $190(15.3 \%)$ \\
\hline Germany 2009 [29] & 1916 & $(16 \%)$ \\
\hline China 2003 [38] & 160 & $28(17.5 \%)$ \\
\hline Italy 2001 [40] & 655 & $89(13.6 \%)$ \\
\hline France 2000 [16] & 125 & $19(15.2 \%)$ \\
\hline Switzerland 1998 [17] & 558 & $151(27 \%)$ \\
\hline Australia 1994 [18] & 245 & $37(15 \%)$ \\
\hline USA 1998 [17] & 1328 & $138(10.4 \%)$ \\
\hline Holland 1992 [13] & 865 & $182(20 \%)$ \\
\hline Japan 1997 [11] & 551 & $165(29.9 \%)$ \\
\hline Italy 1996 [8] & 1417 & $228(16.1 \%)$ \\
\hline Finland 1994 [18] & 1122 & $191(17 \%)$ \\
\hline Finland 1975 [19] & 653 & $89(13.6 \%)$ \\
\hline USA 1962 [1] & & 14 \\
\hline \multicolumn{3}{|c|}{ Developing countries } \\
\hline Study & Total number & Elderly patients percentile \\
\hline Lebanon 2014 [21] & 209 & $18(9 \%)$ \\
\hline Saudi Arabia 2009 [26] & 488 & $50(10.2 \%)$ \\
\hline Colombia 2009 [25] & 693 & $55(7.9 \%)$ \\
\hline Turkey 2008 [30] & 761 & $50(6.6 \%)$ \\
\hline Tunisia 2007 [32] & 219 & $36(7.6 \%)$ \\
\hline Saudi Arabia 2002 [39] & 200 & $18(9 \%)$ \\
\hline India 2009 [44] & 1273 & $82(6.4 \%)$ \\
\hline Nigeria 1977 [15] & 1987 & $60(3.0 \%)$ \\
\hline Lebanon 2014 [21] & 209 & $18(9 \%)$ \\
\hline Saudi Arabia 2009 [26] & 488 & $50(10.2 \%)$ \\
\hline Colombia 2009 [25] & 693 & $55(7.9 \%)$ \\
\hline Turkey 2008 [30] & 761 & $50(6.6 \%)$ \\
\hline
\end{tabular}

based their diagnosis of HSV, VZV, and CMV anterior uveitis on careful ocular and medical history in combination with positive antibody titers or DNA detection in the intraocular fluid using PCR method [17, 21, 25, 34, 42, 43]. However, two studies based their diagnosis of herpetic and CMV uveitis on the clinical findings only $[26,39]$. Ocular tuberculosis, toxoplasmosis, birdshot, and lymphoma were all reported in the elderly population after the year 2000. That might be due to the increased reporting from developing countries after the turn of the century or to increased awareness and advances in diagnostic modalities of some conditions such as ocular tuberculosis and lymphoma (Table 4).

Interestingly, none of the available epidemiology studies found that masquerades or neoplasm was a common cause of uveitis among elderly patients. In addition, only 4 of the reviewed papers reported neoplasms as a cause of uveitis in this age group (12 out of 261 cases) $[10,15,18,20]$. Primary or metastatic ocular lymphoma can present with wide spectrum of age distribution, including young patients [4649]. Actually, our current epidemiology studies demonstrated that ocular lymphoma was one of the common etiologies of ocular inflammation in only one study [16]. As the disease progresses, it can mimic the inflammation of uveitis and is often inappropriately treated with corticosteroids. In primacy intraocular lymphoma, definitive diagnosis requires identification of malignant lymphoid cells from ocular tissue or CSF. Several techniques exist to obtain the required tissue, including aqueous aspiration, diagnostic vitrectomy, and diagnostic retinal or choroidal biopsy [46-49].

Only one study reported the role of diagnostic pars plana vitrectomy (PPV) in our currently reviewed epidemiology reports. Chatzistefanou et al. showed that, of 19 cases that underwent diagnostic PPV, 2 cases had ocular lymphoma and 3 cases were diagnosed with intraocular infection [17]. In addition, many reports previously demonstrated the importance of diagnostic PPV in cases of uveitis with unknown etiology. It was shown that PPV is a helpful tool with diagnostic yield ranging from $14.3 \%$ to $61.5 \%$ of uveitis with unknown causes [50-58].

3.3. Associated Comorbidities. Similarly to the younger patients, autoimmune diseases like sarcoidosis, inflammatory bowel diseases, and insulin dependent diabetes mellitus were reported as common comorbidities among the elderly patients with uveitis [20]. Noninsulin dependent diabetes mellitus was listed by some reports as a common comorbidity among elderly patients with uveitis [17, 20]. However, further data analysis by Chatzistefanou et al. failed to reproduce any specific correlation between diabetes type II and uveitis among the elderly in their study [17].

\section{Discussion}

4.1. Immune System Changes among the Elderly. Uveitis is an inflammatory process affecting one or more of the eye globe layers. Understanding the mechanism of work of the immune system and the changes associated with age is key to explaining the differences observed in uveitis demographics among the elderly patients. It is known that the elements of the innate and the acquired immune system undergo changes with age. This process is labeled immunosenescence [59]. Studies suggest that lymphocytes' ability for proliferation and activation is decreased with age. T-cells among other elements of the immune system are believed to play a major role in ocular inflammatory processes. In particular, Th1 mediated response by T-cells gets altered with age secondary to irregular cell-cell interactions that take place at many levels including the antigen presenting cells (APC) $[2,59]$. This explains the increased rate of infection by certain pathogens in the elderly, like influenza, Herpes, and tuberculosis [60, 61]. As for the paradoxical increase in the number of antibodies produced by B-cells, they are found to be less functional and less specific [59]. The weaker immune system among the elderly renders them more prone to develop uveitis secondary to infectious causes. This was obviously translated in our paper by an increase in the proportion of patients diagnosed 
TABLE 3: Most common location of uveitis in elderly patients.

\begin{tabular}{|c|c|c|c|c|}
\hline Study name $(n)$ & Anterior (\%) & Posterior (\%) & Panuveitis (\%) & Intermediate (\%) \\
\hline Lebanon $2014(n=18)[21]$ & $9(50)$ & $1(5.5)$ & $8(4.5)$ & 0 \\
\hline UK 1994 (71) [20] & $44(62)$ & $7(9.9)$ & $14(19.7)$ & $6(8.5)$ \\
\hline Saudi Arabia $2009(n=50)[26]$ & $39(78)$ & $2(4)$ & $5(10)$ & $4(8)$ \\
\hline Finland $1994(n=191)[18]$ & $187(97.9)$ & $1(0.1)$ & $2(1.04)$ & $1(0.1)$ \\
\hline France $2000(n=19)[16]$ & $5(26.3)$ & $6(31.6)$ & $8(42.1)$ & 0 \\
\hline Japan $2005(n=82)[42]$ & $30(36.6)$ & $24(29.2)$ & $16(19.5)$ & $2(2.4)$ \\
\hline France $2003(n=80)[43]$ & $34(42.5)$ & $18(22.5)$ & $20(25)$ & $8(10)$ \\
\hline Saudi Arabia $2002(n=20)$ [39] & $13(72.2)$ & $2(11.1)$ & $2(11.1)$ & $1(5.6)$ \\
\hline Italy $2010(n=206)[23]$ & $100(48.6)$ & $48(23.3)$ & $54(26.2)$ & $4(19.4)$ \\
\hline Finland $1975(n=86)[19]$ & $80(93)$ & $3(3.4)$ & $3(3.4)$ & 0 \\
\hline Total & 541 & 112 & 129 & 26 \\
\hline
\end{tabular}

TABLE 4: Most common diagnosis of uveitis in elderly patients.

\begin{tabular}{lccc}
\hline Study & Most common & Second common & Third common \\
\hline Lebanon 2014 [21] & Idiopathic uveitis $(5 / 18)$ & HSV $(5 / 18)$ & TB $(3 / 18)$ \\
Colombia 2009 [25] & Toxoplasmosis $(10 / 55)$ & Idiopathic uveitis $(9 / 55)$ & HSV $(5 / 55)$ \\
Saudi Arabia 2009 [26] & AAU $(14 / 51)$ & HSV $(8 / 51)$ & TB $(7 / 51)$ \\
Turkey 2005 [34] & Idiopathic uveitis $(34 / 50)$ & HSV (3/50) & Sarcoidosis $(2 / 50)$ \\
Japan 2005 [42] & Idiopathic uveitis $(53 / 82)$ & HSV/VZV $(10 / 80)$ & HSV (7/82) \\
France 2003 [43] & Idiopathic uveitis $(40 / 80)$ & TB (5/18) & HSV (3/18) \\
Saudi Arabia 2002 [39] & AAU (5/18) & Sarcoidosis $(3 / 19)$ & Lymphoma $(2 / 19)$ \\
France 2000 [16] & Idiopathic uveitis $(5 / 19)$ & HSV $(16 / 138)$ & Sarcoidosis $(11 / 138)$ \\
USA 1998 [17] & Idiopathic uveitis $(43 / 138)$ & Idiopathic uveitis $(21 / 191)$ & Sarcoidosis $(2 / 191)$ \\
Finland 1994 [18] $(n=191)$ & AAU (118/191) & IDDM 5/71 & Sarcoidosis $(3 / 71)$ \\
UK 1994 [20] & Idiopathic uveitis $(55 / 71)$ &
\end{tabular}

AAU: acute anterior uveitis, HSV: herpetic simplex virus, TB: tuberculosis, VZV: Varicella Zoster virus, IDDM: insulin dependent diabetes mellitus.

with herpetic uveitis. It should be noted also that a weak immune reaction in the elderly alters the classical clinical presentation of a disease. Patients with Varicella Zoster ophthalmicus might not manifest the typical skin vesicles. Similarly, endophthalmitis in elderly might not develop a severe inflammatory reaction except late in the course of the disease.

4.2. Interpretation of Our Results. In our study, we have found that the proportion of elderly (aging 60 years or more) among the uveitis patients is more in the developed countries (18.6\%) compared to the developing countries (7.2\%). This observation can be mainly explained by the increasingly aging populations of the developed countries compared to the younger societies in the developing countries [62]. Even with the increased reporting from the developing countries after the year of 2000, the proportion of the elderly among the uveitis patients in these areas did not exceed $8.5 \%$. This suggests that the fewer number of reports published in the developing countries is not responsible for the difference mentioned above between the developing countries and the developed countries.

Extrapolating from the bigger proportion of elderly among the uveitis patients in the developed countries, it is expected that the mean age at presentation of patients with uveitis should be more in those countries compared to the developing countries. In our paper, we found a mean age of presentation of 40.6 in the developed countries, compared to 34.4 in the developing countries.

In our paper, we report that de novo uveitis attacks among the elderly have a female predominance. However female predominance was not always reproducible in all the reports included in our review.

It was also mentioned in our statistics that neoplasms are uncommonly reported. It should be noted however that masquerades occur more frequently among the elderly population and in case they are suspected, an extensive workup is sometimes required in order to rule them out including PPV and tissue analysis [63].

4.3. Diagnosing Uveitis among the Elderly. Uveitis affects all age groups, but the differential diagnosis steers toward specific entities with each age category. Seronegative spondyloarthropathies very rarely manifest as uveitis de novo in elderly patients. On the other hand, masquerades are more common among the elderly and high level of suspicion should be kept in mind while ruling them out.

Location of uveitis is the main subcategory used to differentiate the major entities of uveitis. Clinicopathologic picture, onset and course of the disease, signs and symptoms, 


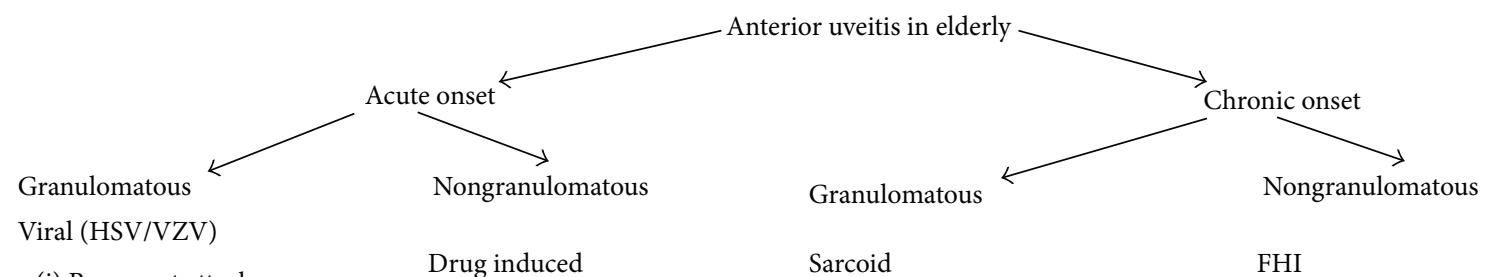

(i) Recurrent attacks

Drug induced

Sarcoid

(i) Rifabutin (sterile hypopyon)

(ii) Elevated IOP

(iii) Fine diffuse keratic precipitates (ii) Cidofovir (i) Pulmonary symptoms

(ii) Cutaneous granuloma

Syphilis

(i) Painless chancre

(ii) Maculopapular rash (palms and soles)

(iii) Skin and mucous membrane gumma

Tuberculosis

(i) Pulmonary symptoms

(ii) Fever, night sweats, and weight loss

Sympathetic ophthalmia

History of ocular surgery/trauma

Fungal/helminthic (i) Heterochromia

(ii) Iris atrophy
SLE
(i) Rash (discoid/malar)
(ii) Photosensitivity
(iii) Arthritis
(iv) Mucosal ulcer
Ulcerative colitis
Bloody diarrhea

Wegener's granulomatosis

(i) Upper respiratory tract abnormalities

(ii) Glomerulonephritis

Ocular ischemic syndrome

Carotid disease

Figure 1: Differential diagnosis of anterior uveitis in elderly patients. HSV: Herpes Simplex virus. VZV: Varicella Zoster virus. VKH: Vogt Kayanagi Harada syndrome. FHI: Fuch's heterochromic iridocyclitis. SLE: Systemic Lupus Erythematosus.

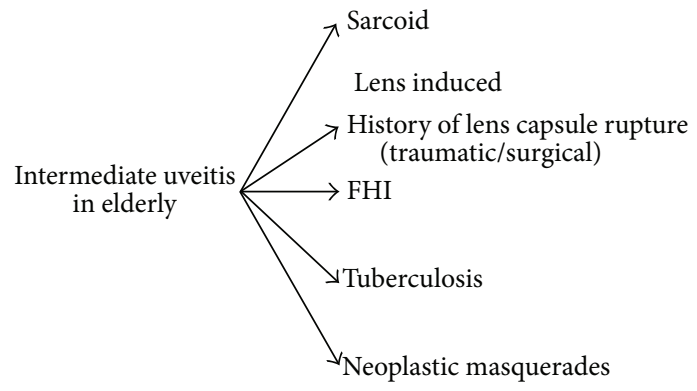

FHI: Fuch's heterochromic iridocyclitis

FIGURE 2: Differential diagnosis of intermediate uveitis in elderly patients. FHI: Fuch's heterochromic iridocyclitis.

and review of system are other useful categories that are employed to narrow down the differential diagnosis.

Figure 1 depicts the entities that would present as anterior uveitis in the elderly. Segregation was based on the onset of the disease, presence or absence of granulomatous reactions, and some distinguishing feature. Special attention should be given to ischemic syndrome secondary to carotid disease in the elderly. Furthermore, viral anterior uveitis is a significant entity in this population. The altered immunologic status of the elderly, generally characterized by a relative cellular immune deficiency, may mask some of the clinical findings warranting definitive diagnostic workup in suspected cases of HSV, VZV, and CMV uveitis using viral PCR of the aqueous humor tap. Figure 2 lists the limited entities of intermediate uveitis in the elderly. It should be noted that pars planitis and multiple sclerosis are not part of the differential listed because these entities are rarely reported in patients older than 60 years of age. It is also worth noting that lens/IOL-induced uveitis is much more common in the elderly cataractous lens. Fuch's heterochromic iridocyclitis and lens-induced uveitis may present as intermediate or as panuveitis. Figure 3 delineates the entities that present as de novo posterior uveitis in the elderly. Many of them are characterized by an extensive inflammation and may present as panuveitis like sympathetic ophthalmia and birdshot chorioretinopathy. Of particular interest, endophthalmitis is more common among the elderly. It presents as panuveitis. One must keep in mind that immunocompromised elderly have a weaker immune system and hence a milder form of inflammation should be anticipated. Sarcoidosis, tuberculosis, and syphilis can mimic any form of uveitis, including panuveitis (Figures 13). Furthermore, in elderly patients with altered immunologic 


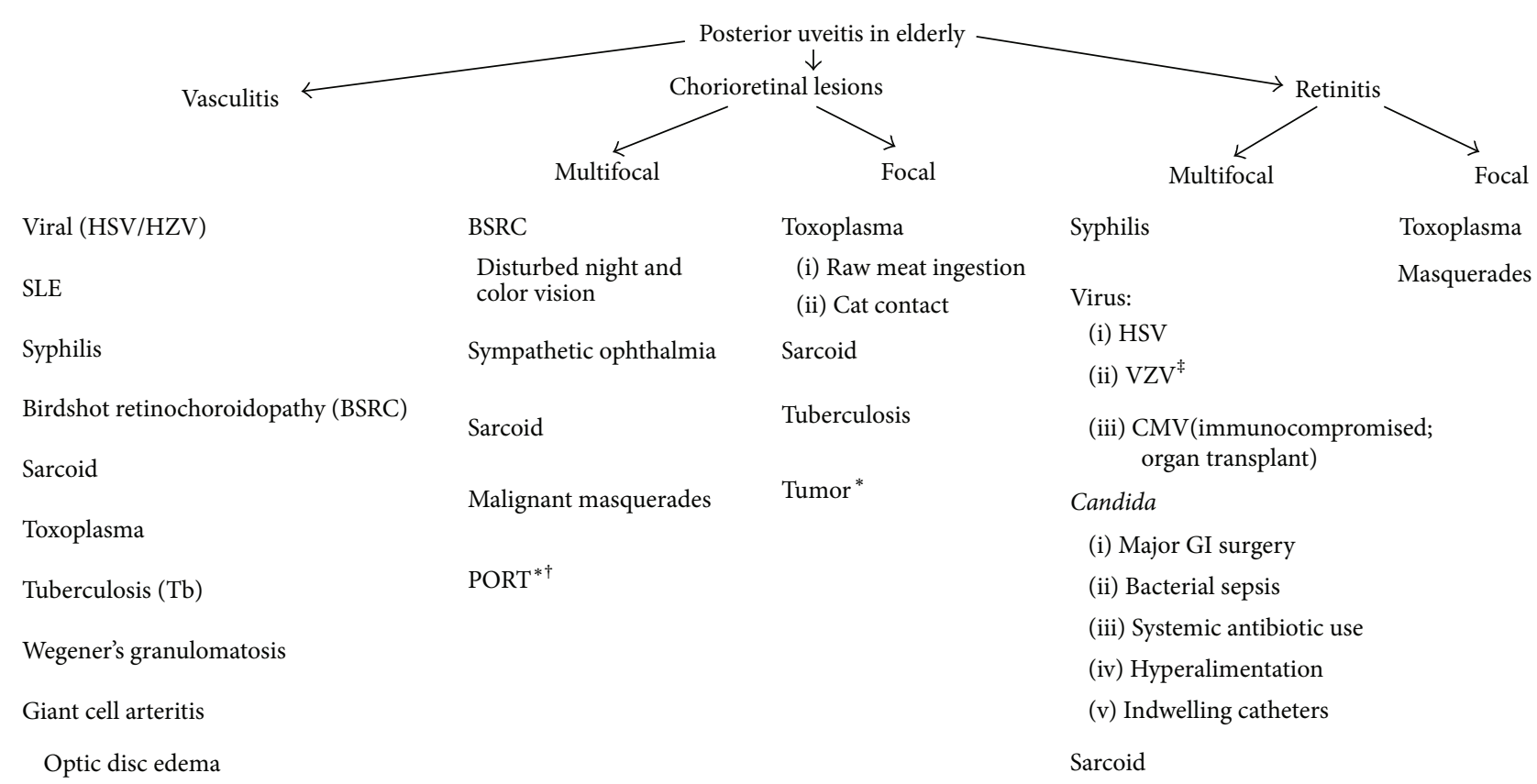

FIGURE 3: Differential diagnosis of posterior uveitis in elderly patients. usually occur $*$ without vitritis. ${ }^{\dagger}$ Elderly and immunocompromised elderly may manifest atypical lesions (large, multiple, and bilateral). ${ }^{\ddagger}$ Immunocompromised elderly may get progressive outer retinal necrosis (PORN). PORT: punctate outer retinal toxoplasmosis. CMV: Cytomegalovirus.

status which may mask some of the clinical findings and with the availability of the less invasive 25 -gauge vitrectomy, diagnostic PPV may be very helpful in determining the unknown etiology of uveitis in the elderly particularly ruling out infections or cancers.

4.4. Treating Uveitis among the Elderly. As in uveitis of any age, treatment is directed to the specific etiology. In case of an infectious etiology, specific anti-infectious medications are given according to the specific organism. While in cases of inflammatory/autoimmune uveitis, treatment with corticosteroids and immune suppressive therapy is instituted. On the other hand, malignant conditions such as lymphoma are treated with local/systemic chemotherapy. However, care should be taken in this elderly population to monitor for drug side effects especially hepatic and renal toxicity and bone marrow suppression, as this age group tends to have other comorbidities and chronic diseases such as osteoporosis, diabetes, and hypertension that puts them at added risks of drug induced complications. Furthermore, drug interactions should be taken into account given the higher likelihood in this age group that the patient will be on medications for other diseases.

\section{Conclusion}

Uveitis in the elderly represents $14.7 \%$ of the total uveitis population reported worldwide. It is more commonly reported in the developing world representing $18.6 \%$ of the uveitis population. The percentage of elderly uveitis patients is increasing with recent reports after the year 2000. Moreover, there is a female preponderance in the elderly uveitis group; however, this ratio is decreasing with recent reports. Anterior uveitis is the most common presentation worldwide and infections such as tuberculosis and toxoplasmosis are increasingly being reported in recent years. Furthermore, neoplasms do not constitute a sizeable proportion of uveitis in the elderly but need to be ruled out as a masquerade syndrome in this age group.

The socioeconomic burden of this disease relies on the fact that it affects mainly the population of productive ages (aging 20 to 60) [64]. Little is mentioned about uveitis as being a cause of blindness among the elderly. But more recently, it was reported that it is underestimated and that such a rare disease among the elderly has a lot of socioeconomic impact especially with the continuously aging societies in the developed countries [65].

\section{Conflict of Interests}

The authors declare that there is no conflict of interests regarding the publication of this paper.

\section{References}

[1] R. W. Darrell, H. P. Wagener, and L. T. Kurland, "Epidemiology of uveitis. Incidence and prevalence in a small urban community," Archives of Ophthalmology, vol. 68, pp. 502-514, 1962.

[2] R. B. Nussenblatt, “The natural history of uveitis," International Ophthalmology, vol. 14, no. 5-6, pp. 303-308, 1990.

[3] L. Dandona, R. Dandona, R. K. John, C. A. McCarty, and G. N. Rao, "Population based assessment of uveitis in an urban 
population in southern India," British Journal of Ophthalmology, vol. 84, no. 7, pp. 706-709, 2000.

[4] D. C. Gritz and I. G. Wong, "Incidence and prevalence of uveitis in Northern California: the Northern California epidemiology of uveitis study," Ophthalmology, vol. 111, no. 3, pp. 491-500, 2004.

[5] D.-K. Hwang, Y.-J. Chou, C.-Y. Pu, and P. Chou, "Epidemiology of uveitis among the Chinese population in Taiwan: a population-based study," Ophthalmology, vol. 119, no. 11, pp. 2371-2376, 2012.

[6] United Nations, Department of Economic and Social Affairs, and Population Division, World Population Ageing 2013, ST/ESA/SER.A/348, 2013.

[7] J. Deschenes, P. I. Murray, N. A. Rao, and R. B. Nussenblatt, "International Uveitis Study Group (IUSG): clinical classification of uveitis," Ocular Immunology \& Inflammation, vol. 16, no. 1-2, pp. 1-2, 2008.

[8] P. Pivetti-Pezzi, M. Accorinti, M. La Cava, R. A. M. C. Gisoldi, and M. A. Abdulaziz, "Endogenous uveitis: an analysis of 1,417 cases," Ophthalmologica, vol. 210, no. 4, pp. 234-238, 1996.

[9] L. H. Thean, J. Thompson, and A. R. Rosenthal, "A uveitis register at the leicester royal infirmary," Ophthalmic Epidemiology, vol. 3, no. 3, pp. 151-158, 1996.

[10] M. J. H. Ronday, J. S. Stilma, R. F. Barbe et al., "Aetiology of uveitis in Sierra Leone, west Africa," British Journal of Ophthalmology, vol. 80, no. 11, pp. 956-961, 1996.

[11] S. Kotake, N. Furudate, Y. Sasamoto, K. Yoshikawa, C. Goda, and H. Matsuda, "Characteristics of endogenous uveitis in Hokkaido, Japan," Graefe's Archive for Clinical and Experimental Ophthalmology, vol. 235, no. 1, pp. 5-9, 1997.

[12] V. T. Tran, C. Auer, Y. Guez-Crosier, N. Pittet, and C. P. Herbort, "Epidemiology of uveitis in Switzerland," Ocular Immunology and Inflammation, vol. 2, no. 3, pp. 169-176, 1994.

[13] A. Rothova, H. J. Buitenhuis, C. Meenken et al., "Uveitis and systemic disease," British Journal of Ophthalmology, vol. 76, no. 3, pp. 137-141, 1992.

[14] J. Palmares, M. F. Coutinho, and J. Castro-Correia, "Uveitis in northern Portugal," Current Eye Research, vol. 9, supplement, pp. 31-34, 1990.

[15] J. O. Ayanru, "The problem of uveitis in Bendel State of Nigeria: experience in Benin City," British Journal of Ophthalmology, vol. 61, no. 10, pp. 655-659, 1977.

[16] L. Bouillet, F. Sarrot Reynauld, B. Gonzalvez, M. Mouillon, J. P. Romanet, and C. Massot, "Uveitis after the age of 60," La Revue de Médecine Interne, vol. 21, no. 12, pp. 1131-1132, 2000.

[17] K. Chatzistefanou, N. N. Markomichelakis, W. Christen, M. Soheilian, and C. S. Foster, "Characteristics of uveitis presenting for the first time in the elderly," Ophthalmology, vol. 105, no. 2, pp. 347-352, 1998.

[18] T. Päivönsalo-Hietanen, H. Vaahtoranta-Lehtonen, J. Tuominen, and K. M. Saari, "Uveitis survey at the University Eye Clinic in Turku," Acta Ophthalmologica, vol. 72, no. 4, pp. 505-512, 1994.

[19] M. Saari, R. Miettinen, and H. Alanko, "Uveitis: report of a 10 year survey in Northern Finland," Canadian Journal of Ophthalmology, vol. 10, no. 3, pp. 356-360, 1975.

[20] K. Barton, C. E. Pavesio, H. M. A. Towler, and S. Lightman, "Uveitis presenting de novo in the elderly," Eye, vol. 8, part 3, pp. 288-291, 1994.

[21] M. Abdulaal, R. Antonios, A. Barikian, M. Jaroudi, and R. N. Hamam, "Etiology and clinical features of ocular inflammatory diseases in a tertiary center in Lebanon," Ocular Immunology \& Inflammation, 2014.

[22] F. Kianersi, Z. Mohammadi, H. Ghanbari, S. M. Ghoreyshi, H. Karimzadeh, and M. Soheilian, "Clinical patterns of uveitis in an Iranian tertiary eye-care center," Ocular Immunology and Inflammation, 2014.

[23] L. Cimino, R. Aldigeri, C. Salvarani et al., "The causes of uveitis in a referral centre of Northern Italy," International Ophthalmology, vol. 30, no. 5, pp. 521-529, 2010.

[24] H. S. Al-Mezaine, D. Kangave, and A. M. Abu El-Asrar, "Patterns of uveitis in patients admitted to a university hospital in Riyadh, Saudi Arabia," Ocular Immunology and Inflammation, vol. 18, no. 6, pp. 424-431, 2010.

[25] A. de-la-Torre, C. A. López-Castillo, J. C. Rueda, R. D. Mantilla, J. E. Gómez-Marín, and J.-M. Anaya, "Clinical patterns of uveitis in two ophthalmology centres in Bogota, Colombia," Clinical and Experimental Ophthalmology, vol. 37, no. 5, pp. 458-466, 2009.

[26] I. H. Hamade, N. Elkum, and K. F. Tabbara, "Causes of uveitis at a referral center in Saudi Arabia," Ocular Immunology and Inflammation, vol. 17, no. 1, pp. 11-16, 2009.

[27] H. Keino, C. Nakashima, T. Watanabe et al., "Frequency and clinical features of intraocular inflammation in Tokyo," Clinical and Experimental Ophthalmology, vol. 37, no. 6, pp. 595-601, 2009.

[28] H. Kitamei, N. Kitaichi, K. Namba et al., "Clinical features of intraocular inflammation in Hokkaido, Japan," Acta Ophthalmologica, vol. 87, no. 4, pp. 424-428, 2009.

[29] E. Jakob, M. S. Reuland, F. Mackensen et al., "Uveitis subtypes in a German interdisciplinary uveitis center-analysis of 1916 patients," Journal of Rheumatology, vol. 36, no. 1, pp. 127-136, 2009.

[30] H. Kazokoglu, S. Onal, I. Tugal-Tutkun et al., "Demographic and clinical features of uveitis in tertiary centers in Turkey," Ophthalmic Epidemiology, vol. 15, no. 5, pp. 285-293, 2008.

[31] K. Pathanapitoon, P. Kunavisarut, S. Ausayakhun, W. Sirirungsi, and A. Rothova, "Uveitis in a tertiary ophthalmology centre in Thailand," British Journal of Ophthalmology, vol. 92, no. 4, pp. 474-478, 2008.

[32] M. Khairallah, S. B. Yahia, A. Ladjimi et al., "Pattern of uveitis in a referral centre in Tunisia, North Africa," Eye, vol. 21, no. 1, pp. 33-39, 2007.

[33] P. Yang, Z. Zhang, H. Zhou et al., "Clinical patterns and characteristics of uveitis in a tertiary center for uveitis in China," Current Eye Research, vol. 30, no. 11, pp. 943-948, 2005.

[34] A. Şengün, R. Karadağ, A. Karakurt, M. S. Saricaoğlu, O. Abdik, and H. Hasiripi, "Causes of uveitis in a referral hospital in Ankara, Turkey," Ocular Immunology and Inflammation, vol. 13, no. 1, pp. 45-50, 2005.

[35] M. Soheilian, K. Heidari, S. Yazdani, M. Shahsavari, H. Ahmadieh, and M. H. Dehghan, "Patterns of uveitis in a tertiary eye care center in Iran," Ocular Immunology and Inflammation, vol. 12, no. 4, pp. 297-310, 2004.

[36] T. Wakabayashi, Y. Morimura, Y. Miyamoto, and A. A. Okada, "Changing patterns of intraocular inflammatory disease in Japan," Ocular Immunology and Inflammation, vol. 11, no. 4, pp. 277-286, 2003.

[37] S. Oruc, A. D. Kaplan, M. Galen, and H. J. Kaplan, "Uveitis referral pattern in a Midwest University Eye Center," Ocular Immunology \& Inflammation, vol. 11, no. 4, pp. 287-298, 2003. 
[38] L.-C. Chou, S.-J. Sheu, M.-C. Hong et al., "Endogenous uveitis: experiences in Kaohsiung Veterans general hospital," Journal of the Chinese Medical Association, vol. 66, no. 1, pp. 46-50, 2003.

[39] S. M. M. Islam and K. F. Tabbara, "Causes of uveitis at The Eye Center in Saudi Arabia: a retrospective review," Ophthalmic Epidemiology, vol. 9, no. 4, pp. 239-249, 2002.

[40] A. Mercanti, B. Parolini, A. Bonora, Q. Lequaglie, and L. Tomazzoli, "Epidemiology of endogenous uveitis in northeastern Italy. Analysis of 655 new cases," Acta Ophthalmologica Scandinavica, vol. 79, no. 1, pp. 64-68, 2001.

[41] A. Bella-Hiag, C. E. Mvogo, and A. Ellong, "Uveitis: epidemiological aspects at the Hospital Laquintinie de Douala," Ophthalmologica, vol. 215, no. 1, pp. 30-33, 2001.

[42] N. Ikeda, S. Hayasaka, and Y. Hayasaka, "Uveitis and pseudouveitis presenting for the first time in Japanese elderly patients," Ophthalmologica, vol. 219, no. 5, pp. 263-266, 2005.

[43] O. Kirsch, M. Lautier-Frau, M. Labetoulle, H. Offret, and E. Frau, "Characteristics of uveitis presenting de novo in the elderly," Journal Francais d'Ophtalmologie, vol. 26, no. 7, pp. 720724, 2003.

[44] D. Das, H. Bhattacharjee, P. K. Bhattacharyya et al., "Pattern of uveitis in North East India: a tertiary eye care center study," Indian Journal of Ophthalmology, vol. 57, no. 2, pp. 144-146, 2009.

[45] D. Wakefield, I. Dunlop, P. J. McCluskey, and R. Penny, "Uveitis: Aetiology and disease associations in an Australian population," Australian and New Zealand Journal of Ophthalmology, vol. 14, no. 3, pp. 181-187, 1986.

[46] C.-C. Chan, J. L. Rubenstein, S. E. Coupland et al., "Primary vitreoretinal lymphoma: a report from an international primary central nervous system Lymphoma Collaborative group symposium," Oncologist, vol. 16, no. 11, pp. 1589-1599, 2011.

[47] S. D. Levasseur, L. A. Wittenberg, and V. A. White, "Vitreoretinal lymphoma: a 20-year review of incidence, clinical and cytologic features, treatment, and outcomes," JAMA Ophthalmology, vol. 131, no. 1, pp. 50-55, 2013.

[48] T. A. Dolecek, J. M. Propp, N. E. Stroup, and C. Kruchko, "CBTRUS statistical report: primary brain and central nervous system tumors diagnosed in the United States in 2005-2009," Neuro-Oncology, vol. 14, supplement 5, pp. vl-v49, 2012.

[49] States CBTRotU, "Primary brain and central nervous system tumors diagnosed in the United States in 2004-2007," CBTRUS Statistical Report, Central Brain Tumor Registry of the United States, Hinsdale, Ill, USA, 2010.

[50] H. Priem, H. Verbracken, and J. J. De Laey, "Diagnostic problems in chronic vitreous inflammation," Graefe's Archive for Clinical and Experimental Ophthalmology, vol. 231, no. 8, pp. 453-456, 1993.

[51] G. N. Palexas, W. R. Green, M. F. Goldberg et al., "Diagnostic pars plana vitrectomy report of a 21-year retrospective study," Transactions of the American Ophthalmological Society, vol. 93, pp. 281-308, 1995.

[52] H. Verbraeken, "Diagnostic vitrectomy and chronic uveitis," Graefe's Archive for Clinical and Experimental Ophthalmology, vol. 234, no. 1, pp. S2-S7, 1996.

[53] P. Mruthyunjaya, J. M. Jumper, R. McCallum, D. J. Patel, T. A. Cox, and G. J. Jaffe, "Diagnostic yield of vitrectomy in eyes with suspected posterior segment infection or malignancy," Ophthalmology, vol. 109, no. 6, pp. 1123-1129, 2002.

[54] S. E. Coupland, N. E. Bechrakis, G. Anastassiou et al., "Evaluation of vitrectomy specimens and chorioretinal biopsies in the diagnosis of primary intraocular lymphoma in patients with Masquerade syndrome," Graefe's Archive for Clinical and Experimental Ophthalmology, vol. 241, no. 10, pp. 860-870, 2003.

[55] J. L. Davis, D. M. Miller, and P. Ruiz, "Diagnostic testing of vitrectomy specimens," American Journal of Ophthalmology, vol. 140, no. 5, pp. 822-829, 2005.

[56] R. Margolis, O. F. M. Brasil, C. Y. Lowder et al., "Vitrectomy for the diagnosis and management of uveitis of unknown cause," Ophthalmology, vol. 114, no. 10, pp. 1893-1897, 2007.

[57] R. Margolis, "Diagnostic vitrectomy for the diagnosis and management of posterior uveitis of unknown etiology," Current Opinion in Ophthalmology, vol. 19, no. 3, pp. 218-224, 2008.

[58] A. Oahalou, P. A. W. J. F. Schellekens, J. D. de Groot-Mijnes, and A. Rothova, "Diagnostic pars plana vitrectomy and aqueous analyses in patients with uveitis of unknown cause," Retina, vol. 34, no. 1, pp. 108-114, 2014.

[59] S. C. Castle, "Clinical relevance of age-related immune dysfunction," Clinical Infectious Diseases, vol. 31, no. 2, pp. 578-585, 2000.

[60] G. Gavazzi and K.-H. Krause, "Ageing and infection," The Lancet Infectious Diseases, vol. 2, no. 11, pp. 659-666, 2002.

[61] E. Crétel, I. Veen, A. Pierres, P. Bongrand, and G. Gavazzi, "Immunosénescence et infections, mythe ou réalité?" Médecine et Maladies Infectieuses, vol. 40, no. 6, pp. 307-318, 2010.

[62] United Nations Department of Economic and Social Affairs; Population Division, World Population Prospects: The 2012 Revision, Volume II, Demographic Profiles, (ST/ESA/SER.A/345), 2013, World Population Ageing, ST/ESA/SER.A/348, 2013.

[63] P. Zamiri, S. Boyd, and S. Lightman, "Uveitis in the elderlyis it easy to identify the masquerade?" British Journal of Ophthalmology, vol. 81, no. 10, pp. 827-831, 1997.

[64] M. S. A. Suttorp-Schulten and A. Rothova, "The possible impact of uveitis in blindness: a literature survey," The British Journal of Ophthalmology, vol. 80, no. 9, pp. 844-848, 1996.

[65] S. W. Reeves, F. A. Sloan, P. P. Lee, L. Van Scoyoc, and G. J. Jaffe, "Uveitis in the elderly: epidemiological data from the national long term care survey medicare cohort," Ophtalmology and Visual Science, vol. 46, no. 5, p. 5011, 2005. 


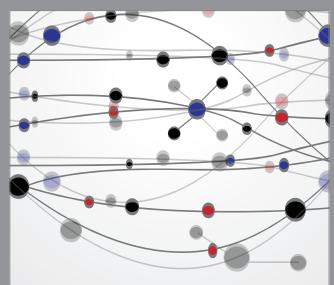

The Scientific World Journal
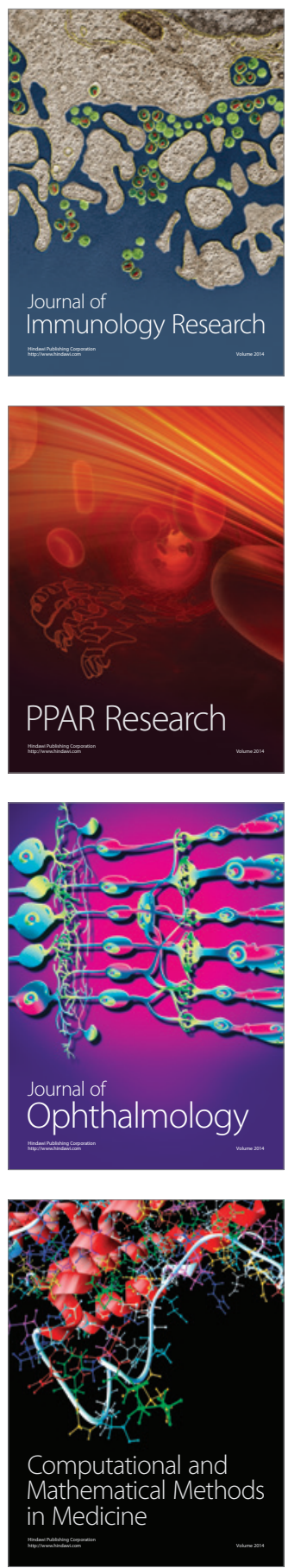

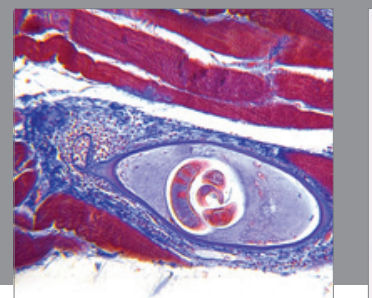

Gastroenterology

Research and Practice
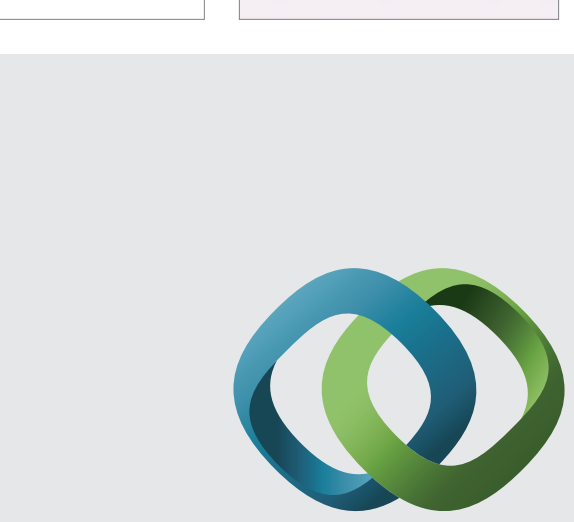

\section{Hindawi}

Submit your manuscripts at

http://www.hindawi.com
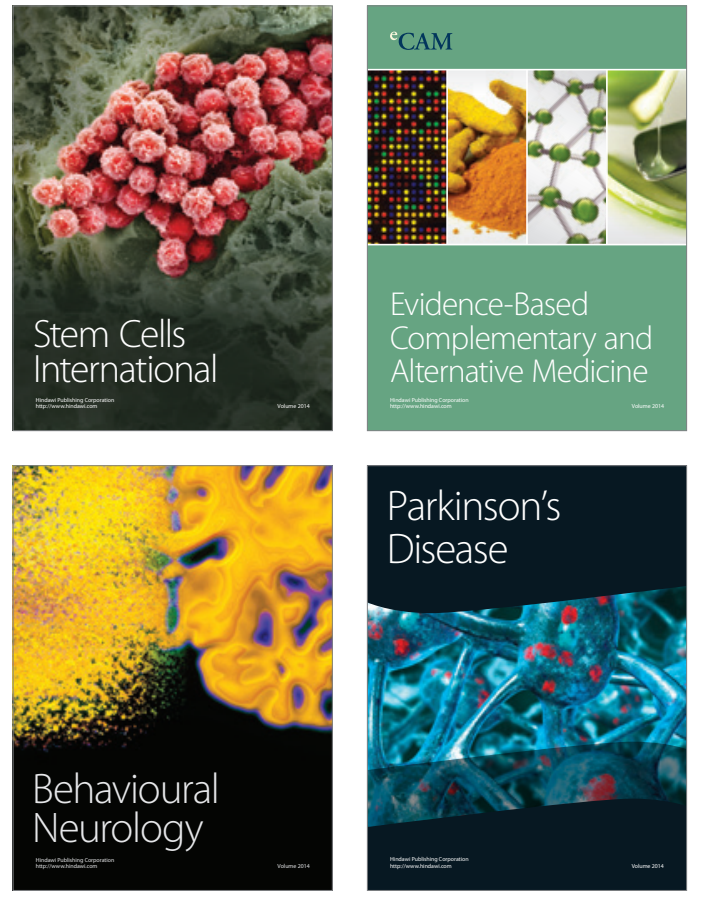
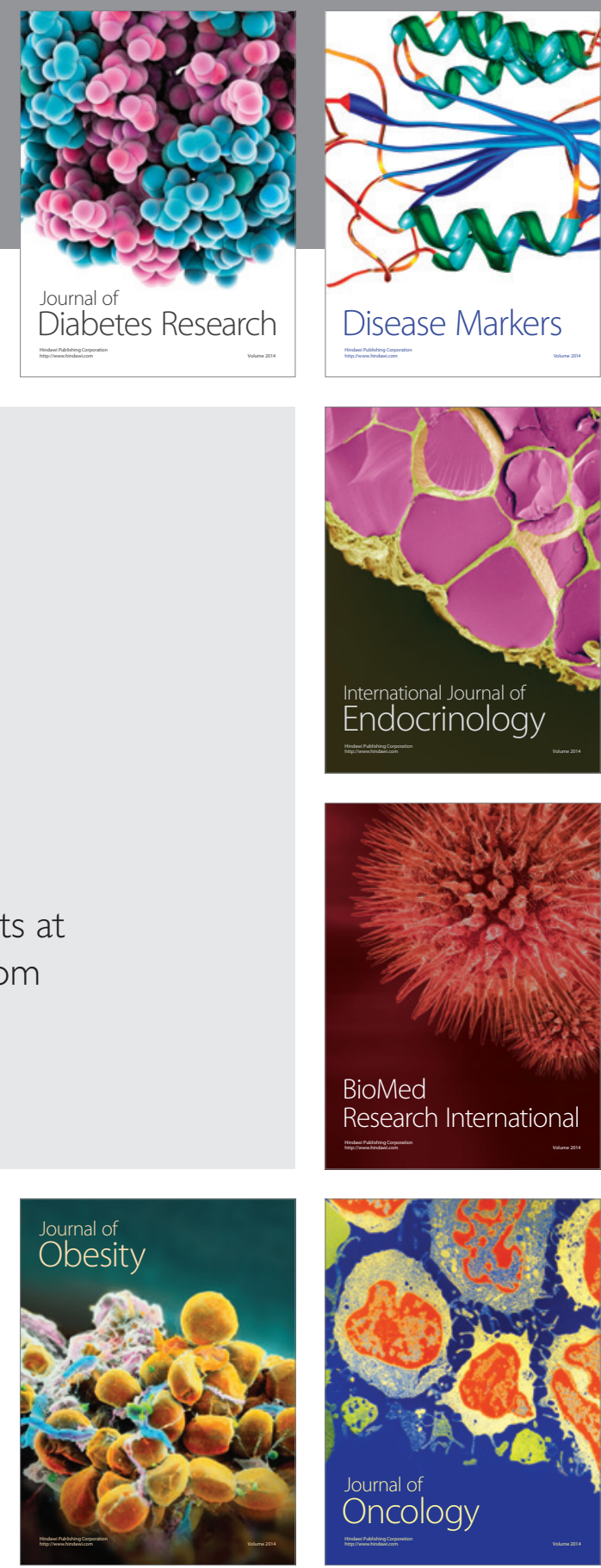

Disease Markers
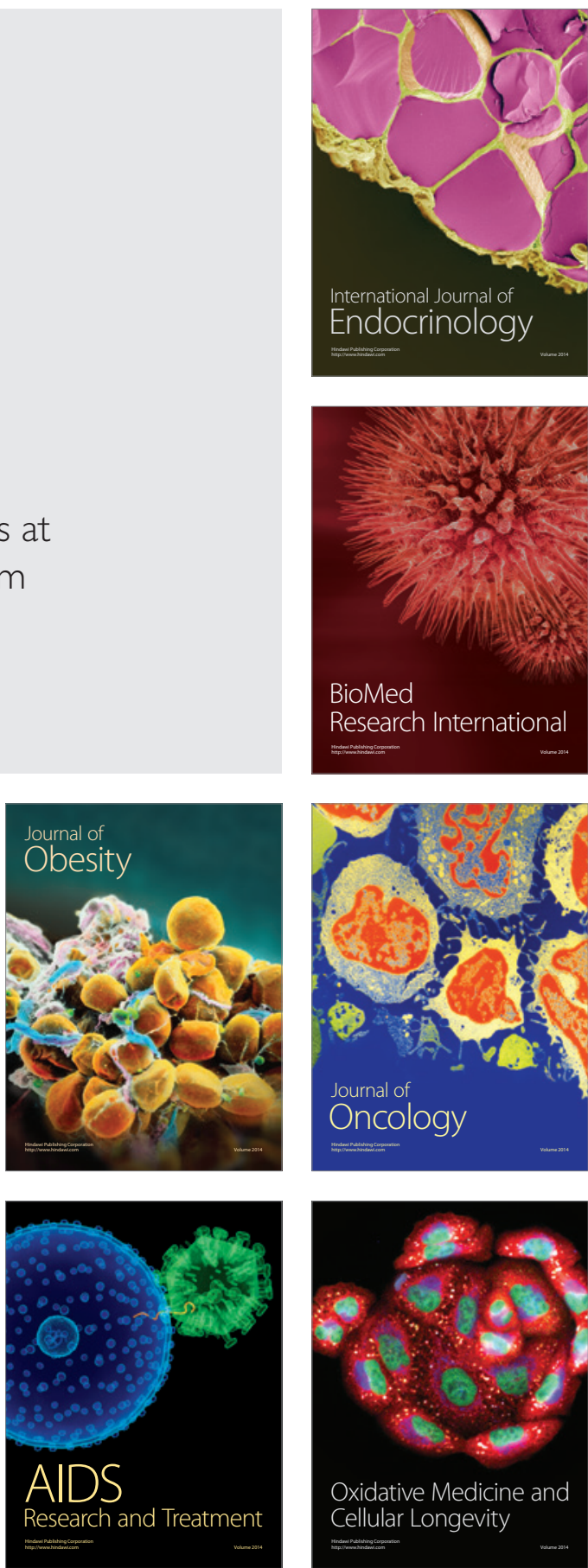